\title{
Movable Thrombi Diagnosed Using Ultrasonography in Non-Significant Carotid Stenosis
}

\author{
Gyihyaon Yun (D), Sung Hyuk Heo (D), Dai-Il Chang (D), Bum Joon Kim (D) \\ Department of Neurology, Kyung Hee University Hospital, Seoul, Korea
}

Stenosis of an artery of more than $50 \%$ is usually regarded as significant stenosis, which may cause ischemic stroke. However, the nature of plaque may be important, even though the stenosis does not reach a significant degree. A 75-year old man with right arm weakness showed multiple scattered small infarct lesions at the left middle cerebral artery territory with mild stenosis of the left carotid artery. No embolic source was detected from cardiac assessments. Carotid duplex sonography showed a mobile plaque at the carotid artery, which was not detected by conventional angiography. Our case report suggests that direct vessel wall imaging can be useful in patients who have ischemic stroke with an embolic pattern without significant arterial stenosis.

J Neurosonol Neuroimag 2019;11(2):145-148

Key Words: Carotid artery ulcerating plaque; Ultrasonography, Doppler, Duplex; Carotid artery thromboses

\author{
Received: June 12, 2019 \\ Revised: July 12, 2019 \\ Accepted: August 3, 2019 \\ Address for correspondence: \\ Bum Joon Kim \\ Department of Neurology, \\ Kyung Hee University Hospital, \\ 23 Kyungheedae-ro, Dongdae- \\ mun-gu, Seoul 02447, Korea \\ Tel: $+82-2-958-8499$ \\ Fax: $+82-2-958-8490$ \\ E-mail: medicj8o@hanmail. \\ net
}

Ischemic stroke can be caused by various mechanisms and secondary prevention strategies focus on the mechanisms of stroke. Therefore, ischemic stroke patients receive extensive evaluations to determine the exact stroke mechanism. These evaluations include cerebral artery angiography by magnetic resonance imaging (MRI), computed tomography, or digital subtraction angiography (DSA). Usually, $>50 \%$ stenosis is regarded as significant, which may be responsible for an ischemic lesion in the corresponding brain area. If there is no significant stenosis, additional workups to detect the cardioembolic source or coagulopathies are performed. If the cause of stroke is still not found after extensive evaluation, stroke is regarded as an embolic stroke of unknown source (ESUS). ${ }^{1}$

Here, we report a case of embolic infarction without significant stenosis at the proximal artery and a mobile plaque observed by carotid duplex ultrasonography. Our case may show the usefulness of carotid ultrasonography in patients who have ischemic stroke with an embolic pattern without significant arterial stenosis.

\section{CASE REPORT}

A 75-year-old hypertensive male patient visited Stroke Center of Kyung Hee University Hospital with right arm weakness, which occurred the day before. Blood pressure measured at the emergency center was 130/70 $\mathrm{mmHg}$ and pulse rate was 72 beats/min showing normal sinus rhythm. The right-side weakness was motor research council grade 4 with a mild ataxia. Dysarthria was also observed. Multiple high-signal intensity lesions were observed in the left middle cerebral artery (MCA) territory on diffusion-weighted imaging (DWI) (Fig. 1A). Mild stenosis (<50\% diameter reduction) was observed in the left internal carotid artery (ICA) (Fig. 1B). The patient received aspirin (100 mg/day) and clopidogrel ( $75 \mathrm{mg} /$ day) for acute stroke treatment. At 2 and 4 days after admission, dysarthria and right-side weakness progressed and transient aphasia developed. Follow-up DWI demonstrated new scattered lesions in the left MCA territory (Fig. 1C, D). Further evaluation by DSA showed $42 \%$ stenosis without an ulcer or flow disturbance of the ICA (Fig. 1E). Transthoracic 

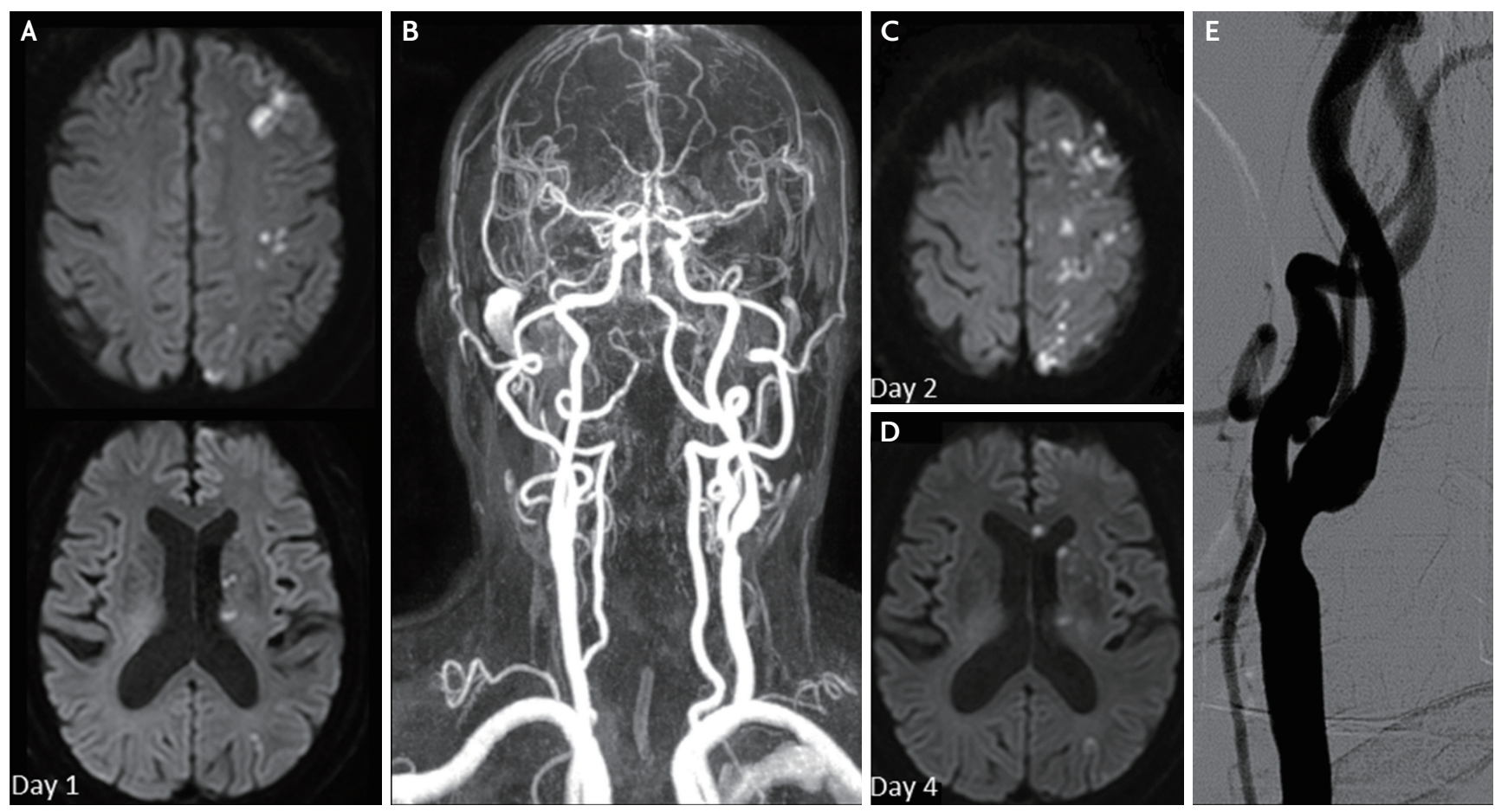

FIG. 1. Magnetic resonance imaging and angiography of cerebral arteries images of the patient. (A) One day after admission, multiple scattered lesions were observed on initial diffusion-weighted image. (B) Mild stenosis ( $<50 \%)$ was observed at the left internal carotid artery (ICA) on contrast-enhanced magnetic resonance angiography. (C, D) New scattered lesions in the left hemisphere at day 2 and 4 after admission.

(E) Intraplaque hemorrhage with a surface ulcer in the left ICA on digital subtraction angiography.

echocardiography showed an enlarged left atrium (anterior-posterior diameter: $50 \mathrm{~mm}$, left ventricular diameter volume index: $35 \mathrm{~mL} / \mathrm{m}^{2}$ ), normal left ventricular systolic function (ejection fraction: $76 \%$, left ventricular end diastolic volume/end-systolic volume: 55/13 mL), and no significant valvular disease. Although 24-hour Holter monitoring was performed to detect paroxysmal atrial fibrillation, arrhythmia was not detected.

To directly evaluate vessel wall pathology, carotid ultrasonography was performed. Stenosis (47\% diameter reduction) was observed with a mobile lesion (Fig. 2A). It was regarded as a ruptured plaque or dissecting flap. From high-resolution MRI (HRMRI), the mobile lesion was more likely a ruptured plaque located adjacent to an atheroma (Fig. 2B, C). Patient maintained dual antiplatelet treatment with high-dose statin, and at the follow-up carotid duplex ultrasonography, 6 months after stroke onset, the mobile plaque disappeared (Fig. 2D).

\section{DISCUSSION}

The current case shows a patient with ischemic stroke that recurred at the same vascular territory within a relatively short period. No significant stenosis or cardioembolic source was observed from extensive examination. An ulcerative plaque or flow disturbance was not observed in the DSA. However, carotid duplex ultrasonography showed a mobile lesion, which was confirmed as a ruptured plaque via HRMRI, with both techniques directly showing the vessel wall pathology.

The patient experienced recurrent ischemic stroke in a single vascular territory and was assumed to have an embolic source in that particular vessel. Therefore, DSA was performed to quantitatively measure the stenosis degree, evaluate the morphology of the stenosis area, and confirm any flow disturbance which might induce platelet activation. However, the DSA results showed that the stenosis degree was non-significant, and no ulcerative lesions or flow disturbance was observed. On the other hand, duplex ultrasonography was helpful in showing the direct vessel wall pathology of stenosis. ${ }^{2}$ 

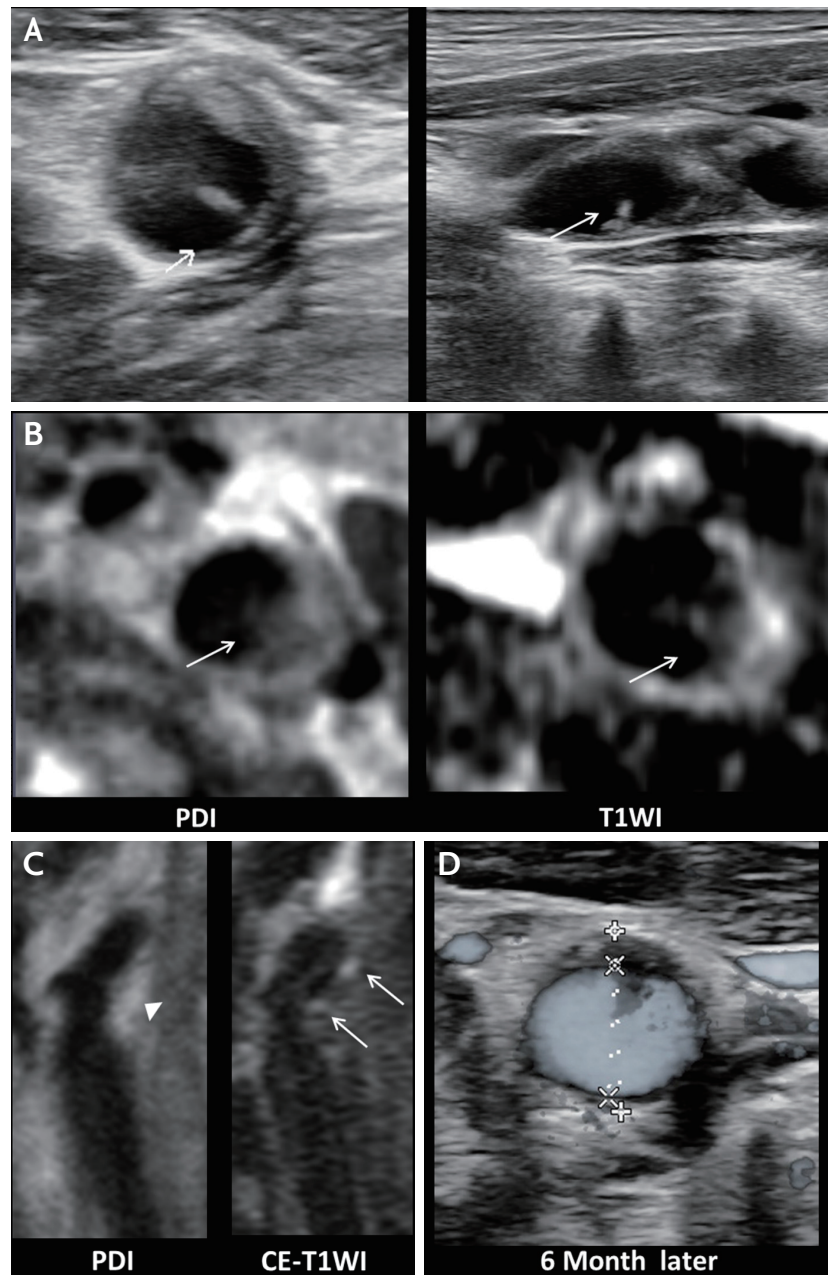

FIG. 2. Carotid duplex and high-resolution magnetic resonance imaging (HRMRI) findings of the patient. (A) Mobile lesion (arrows) and plaque observed from initial carotid duplex ultrasonography. (B) Axial HRMRI images show a mobile lesion at the site of ulcerated plaque (arrows). (C) Coronal high-resolution magnetic resonance images show unstable plaque with intraplaque hemorrhage (arrowhead) and multiple focal enhancement (arrows). (D) Six months after stroke onset, mobile lesion disappeared at the follow-up carotid duplex ultrasonography.

The stenosis degree was still under 50\%, but a mobile lesion was observed. The atheroma showed a heterogeneous echogenicity and the mobile plaque was located at the shoulder region of the atheroma.

Previous studies have differentiated mobile lesions according to the sonographic characteristics. Dissecting flaps were usually observed where the atherosclerotic plaque was not dominant, and the hypoechoic lesions were continuously observed to the distal area with a gradual decrease in the lumen area. ${ }^{3}$ An embolic source from the more proximal area are usually observed as a distinguishable mobile lesion isolated from the underlying atheroma. In our case, the oval-shaped mobile lesion was adjacent to the atheroma with a continuous contour and no ulcerative lesion was observed nearby. ${ }^{4}$ HRMRI showed an intraplaque hemorrhage with a surface ulcer, which supported the identification of the mobile lesion as a ruptured plaque. ${ }^{5}$

The term ESUS was first used to define those who potentially have a cryptogenic cause of the cardioembolic source and benefit from anticoagulation. Recently, new oral anticoagulant (NOAC) trials in ESUS patients have failed due to the heterogeneity of ESUS, ${ }^{6}$ especially in atherothrombotic infarction ESUS patients who may benefit from antiplatelet agents, which may have led to the failure of NOAC trials. ${ }^{7}$ Although the direct imaging of vessel wall using carotid duplex sonography or high-resolution vessel wall MRI may be helpful for excluding patients with non-significant stenosis, it may also exclude a subset of those patients with a potential source of artery-to-artery embolism.

Usually, carotid duplex sonography is not essential for patients with non-significant stenosis. However, for patients with an embolic lesion pattern restricted to a single vascular territory, direct imaging of the vessel wall by carotid duplex ultrasonography may be beneficial for finding a potential artery-to-artery embolic source, even in those with arterial stenosis of a non-significant degree.

\section{Acknowledgments}

This work was supported by the National Research Foundation of Korea (NRF) grant funded by the Korea Government (MSIT; No. 2017R1C1B5017843).

\section{Conflicts of Interest}

No potential conflicts of interest relevant to this article was reported.

\section{REFERENCES}

1. Hart RG, Diener HC, Coutts SB, Easton JD, Granger CB, O'Donnell MJ, et al. Embolic strokes of undetermined source: the case for a new clinical construct. Lancet Neurol. 2014;13:429-438.

2. Brinjikji W, Rabinstein AA, Lanzino G, Murad MH, Wil- 
liamson EE, DeMarco JK, et al. Ultrasound characteristics of symptomatic carotid plaques: a systematic review and meta-analysis. Cerebrovasc Dis. 2015;40:165-174.

3. Benninger DH, Baumgartner RW. Ultrasound diagnosis of cervical artery dissection. Front Neurol Neurosci. 2006;21:7084.

4. Matsumoto K, Hashimura N, Li Z, Nagai T, Matsuda K. Characteristics and therapies of mobile lesions in the carotid artery. Neurosonol. 2016;29:22-27.

5. DeMarco JK, Huston J 3rd. Imaging of high-risk carotid artery plaques: current status and future directions. Neurosurg Focus. 2014;36:E1.

6. Mohr JP, Thompson JL, Lazar RM, Levin B, Sacco RL, Furie KL, et al. A comparison of warfarin and aspirin for the prevention of recurrent ischemic stroke. $N$ Engl J Med. 2001;345:1444-1451.

7. Hart RG, Sharma M, Mundl H, Kasner SE, Bangdiwala SI, Berkowitz SD, et al. Rivaroxaban for stroke prevention after embolic stroke of undetermined source. N Engl J Med. 2018;378:2191-2201. 